\title{
Open Mesh versus Laparoscopic Mesh Repair of Inguinal Hernia : in Terms of Patient Satisfaction
}

\author{
Umesh Singh, Varnika Gupta ${ }^{2}$

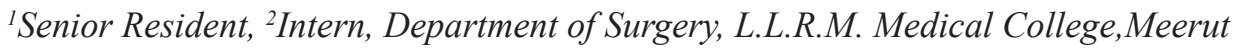

\begin{abstract}
Background- Inguinal hernia is the most common type of external abdominal hernia. Open

Lichtenstein method or laparoscopic method can be employed to repair inguinal hernia. The superiority of laparoscopic mesh hernioplasty over open mesh hernioplasty is debatable.

Method- This prospective study was done on 120 patients in the department of surgery, LLRM medical college, Meerut to evaluate the usefulness of laparoscopic mesh hernioplasty (TEP Technique) for inguinal hernia repair versus conventional open mesh hernioplasty (Lichtenstein Technique) in terms of complications, pain and recurrence. Equal number of subjects were put in two groups : open mesh repair group and laparoscopic mesh repair group.
\end{abstract}

Results- The mean age of the patients with inguinal hernia including both groups was 50 yrs. The mean operation time of Laparoscopic TEP mesh hernioplasty was 71.33 minutes while that of Open Lichtenstein repair was 36.33 minutes. Postoperative analgesic requirement was significantly lower in the patients operated by TEP technique as compared with patients treated by Open Lichtenstein's technique. In TEP group, two patients developed neuralgia, one patient developed fever, 1 patient developed hematoma whereas in the open group there were 3 cases of neuralgia formation and 3 cases of fever.

Conclusion- In all, Laparoscopic mesh hernioplasty using TEP technique is a novel technique with its share of advantages and disadvantages. There are potential benefits of laparoscopic inguinal hernia repair for inguinal hernias in terms of post-operative pain, hospital stay and post operative complications. We recommend that a surgeon should use this laparoscopic technique for hernia repair after knowing all the pros and cons of the technique, his expertise for the technique and the infrastructure available.

Keywords-open mesh, laparoscopic, hernioplasty, mesh,

\section{Introduction}

A hernia is defined as an abnormal protrusion of a viscus or a part of it through the wall that contains it but without a breach in the body. The commonest variety of hernia is the protrusion of abdominal viscera through the abdominal wall. Among these, inguinal hernia is the most common hernia accounting for $75 \%$, incisional and ventral hernias for approx. $10 \%$, femoral hernias for

\section{Corresponding author : \\ Umesh Singh}

Contact number: 9457817225

Address: 224/9 Jagriti Vihar, Meerut, 250004 approx. 3\% and unusual hernias for remaining 5-10\% of all abdominal hernias. Inguinal hernia being the most common type of external abdominal hernia is the matter of our study here

Inguinal hernioplasty has undergone a gradual evolution over the last 100 years. The introduction of video laparoscopy and the development of new laparoscopic instruments and skills offered the potential to take the posterior approach to inguinal hernioplasty one step further and make it less invasive and less disruptive to patients. Initially most laparoscopic surgeons used a transabdominal preperitoneal (TAPP) approach to access the posterior floor of the groin. This approach soon became the standard for laparoscopic hernia 
repair and many studies were published demonstrating recurrence rates of less than $1 \%$. When complications such as internal hernia from inadequate closure of the peritoneum and injury to viscera from trocars and needles placed in the peritoneal cavity were reported, a totally extraperitoneal approach (TEP) was developed and subsequently adopted by many laparoscopic surgeons. This approach required the surgeon to laparoscopically expose the extraperitoneal space without entering the peritoneal cavity.

The present study was conducted to evaluate the usefulness of laparoscopic mesh hernioplasty (TEP Technique) for inguinal hernia repair versus conventional open mesh hernioplasty (Lichtenstein Technique) in terms of learning curve, operative time, complications, hospital stay, wound infection, pain, interference with routine activities, sick leave, recurrence and overall satisfaction.

\section{Materials and Method}

The present study was conducted in the department of surgery, LLRM Medical College, Meerut on randomly selected patients of inguinal hernia, who were admitted and underwent hernia repair which was either laparoscopic repair or open repair in SVBP Hospital, Meerut, from July, 2017 to October, 2018. Total 120 cases of inguinal hernia were studied of which 60 were operated by Laparoscopic technique and the other 60 were operated by Open Lichtenstein's technique. Both direct and indirect hernias were included in the study. The workup of the patient was divided into preoperative, operative and postoperative monitoring and follow up.

Informed consent was taken from all the patients before entering the study. All patients were kept nil per orally (NPO) from midnight before surgery. The operation time from the skin incision to the application of the last stitch was noted. For postoperative pain charting, Visual Analogue scale was explained and discussed with the patient preoperatively.

During the surgery, note was made of any anatomical abnormalities, type of hernia, extent of sac, any intraoperative complication, size of mesh applied , method of fixation of mesh and operative time. All patients were examined on the evening of surgery by the operating surgeon and were examined for general condition and any early post operative complications like early recurrence, bleeding from port site/ hematoma formation, fever, scrotal swelling and cough.
Prophylactic antibiotics were given. Complications like seroma formation, wound infection and fever were noted. Wound of patient was assessed and stitch removal was done accordingly.

\section{Statistical Analysis}

All the data were compiled on Microsoft excel computer program and was subjected to statistical analysis. Student's ' $t$ ' test and Chi square test were used to study the significance of differences of various parameters in laparoscopic TEP and open inguinal hernia group.

\section{Observations and Results}

The peak incidence of hernia was in $41-60 \mathrm{yrs}$ of age group. Mean age of the patients who underwent Laparoscopic mesh hernioplasty was 44.6yrs while mean age of the patients who underwent Open mesh hernioplasty was $48.3 \mathrm{yrs}$. Table 1 shows the mean range and Standard Deviation of age of both age groups. There is no significant difference in the two age groups.

Majority of patients have right sided hernia 62 (51.67\%), 32(53.3\%) were in TEP group and $30(50 \%)$ were in open group. All the patients included in the study are males except 1 patients who was operated by Open mesh hernioplasty with male to female ratio being 59:1.

The mean operation time of Laparoscopic TEP mesh hernioplasty was 71.33 minutes with STANDARD DEVIATION of \pm 23.8 . The mean operative time of Open Lichtenstein repair was 36.33 minutes with a STANDARD DEVIATION of \pm 9.643 .

The postoperative pain was recorded at $12 \mathrm{hrs}, 24$ hrs, 48 hrs and 7 days after the operation by using Visual Analogue Scale (VAS) pain scoring system. The mean pain out of 5 in Laparoscopy group was significantly lower as compared to mean pain score in Open group ( $p$ value $<0.05$ ) except pain scores at 7 days in which there was no significant statistical difference between the two groups $(p-$ value $>0.05)$.(Table 2$)$. The mean amount of analgesic consumed was 2.57 in the TEP group and 6.0 in the open group. The analgesic requirement in the TEP group was significantly less than the open group ( $p$ value $<0.05$ ). Peritoneal breach was an intra-operative complication that occurred in laparoscopic group which occurred in 2 patients, also in 1 patient major vascular injury was found(Table 3). There was no incidence of epigastric artery injury or injury to vas deferens in both 
groups.

In the Lichtenstein group, majority of patients were discharged on days $3-6(58)(96.7 \%)$. On the other hand in the laparoscopic group, majority of patients were discharged on day $1-3(48)(80 \%)$.

There was only one recurrence that was noted during follow up in OPD in the Laparoscopy group and in the Open Lichtenstein group. The patient with recurrence then underwent Open surgery and Lichtenstein repair was done.

In the TEP group, majority of patients needed 7 days sick leave where as those in the Lichtenstein group needed more than 7 days sick leave. In the TEP group, total 4 patients had interference in their daily routine activities whereas in lichtenstein's group total 24 patients had interference in their daily routine activities.

Table 1: Patients Age Profile (Mean, Standard Deviation And Range)

\begin{tabular}{|l|l|l|}
\hline AGE(YEARS) & LAP - TEP & LICHTENSTEIN \\
\hline MEAN & 44.60 & 48.60 \\
\hline RANGE & $18-67$ & $18-90$ \\
\hline STANDARD DEVIATION & \pm 23.6 & \pm 21.3 \\
\hline
\end{tabular}

TABLE 2 :VISUAL ANALOGUE SCALE (VAS) PAIN SCORE MEAN

\begin{tabular}{|l|l|l|l|}
\hline TIME AFTER OPERATION & TEP & LICHTENSTEIN & p VALUE \\
\hline 12 HOURS & 1.5 & 2.43 & 0.0006 \\
\hline 24 HOURS & 1.03 & 2.10 & 0.013 \\
\hline 48 HOURS & 0.5 & 1.83 & 0.018 \\
\hline $7^{\text {TH }}$ DAY & 0.2 & 0.47 & 0.263 \\
\hline
\end{tabular}

TABLE 3 :INTRAOPERATIVE COMPLICATIONS

\begin{tabular}{|l|l|l|}
\hline COMPLICATIONS & TEP (n=60)(\%) & $\begin{array}{l}\text { LICHTENSTEIN } \\
\mathbf{( n = 6 0 ) ( \% )}\end{array}$ \\
\hline PERITONEAL BREACH & $2(3.3 \%)$ & $0(0 \%)$ \\
\hline INFERIOR EPIGASTRIC ARTERIAL INJURY & $0(0 \%)$ & $0(0 \%)$ \\
\hline VAS DEFERENS INJURY & $0(0 \%)$ & $0(0 \%)$ \\
\hline MAJOR VASCULAR INJURY & $1(1.67 \%)$ & $0(0 \%)$ \\
\hline VISCERAL INJURY & $0(0 \%)$ & $0(0 \%)$ \\
\hline NERVE INJURY & $0(0 \%)$ & $0(0 \%)$ \\
\hline
\end{tabular}




\section{Discussion}

The age-wise distribution as reflected in our observation indicates that most of the patients of inguinal hernia in our study belongs to $41-60 \mathrm{yrs}$ of age group. In a study by Fasih and Mahapatra ${ }^{(1)}$ at Barnsley district general hospital, UK (2000), mean age of patients of inguinal hernia was found to be $\mathbf{5 4}$ years (95\% between $46-61$ years). In a study by Franciosi (2) at Milano, in a series of 692 cases of inguinal hernia, mean age was $\mathbf{6 0}$ years (range $18-88$ years). The mean age calculated in our study is lesser as compared to various studies in literature. This is due to the fact that subjects were allowed to chose the procedure and more patients from the younger age group enrolled in the Laparoscopic group.

According to Jonathan Macready, Surgeon to the city of London truss society ${ }^{(3)}$, "The tendency to the production of inguinal hernia in males hardly diminishes at all as age advances. Men are subject to this peculiarity, from which women are almost free..." In our study also there is male preponderance with $99.16 \%$ of cases being males and only $0.83 \%$ of cases being females.

In the present study $62(51.67 \%)$ were right sided,32(26.6\%) were left sided and $26(21.6 \%)$ were bilateral hernias . More or less similar data are reported by various workers in the field. Gross and Snyder ${ }^{(4)}$ reported right sided inguinal hernia 60\%. Left sided hernia $25 \%$ and bilateral hernia $15 \%$. Stoker et al ${ }^{(5)}$ and Liem et al. ${ }^{(6)}$ have shown that operative time correlates inversely with experience.

In our study the mean pain out of 10 according to VAS score in Laparoscopy group was significantly lower as compared to mean pain score in Open group ( $p$ - value $<0.05)$.This was in concordance with the study conducted by Neumayer et al ${ }^{(7)}$ [ 2004] which also reported lesser immediate postoperative pain in comparison of TEP versus Open Lichtenstein's repair.

The complication rate reported for laparoscopic repairs ranges from less than $3 \%{ }^{(8)}$ to as high as $20 \%$, ${ }^{(9)}$ and is similar to that reported for open repairs ${ }^{(10)}$. As discussed earlier, the incidence of complications is directly related to the point on the learning curve studied. The incidence of hydrocele following laparoscopic repair is the same as after open repairs and occurs in less than $1 \%$ of patients. ${ }^{(11)}$

Lal et al ${ }^{(12)}$ in 2003, Wennstrom et al ${ }^{(13)}$ in 2004 and
Neumayer et al (7) in 2004 reported similar recurrence rates for both TEP mesh hernioplasty and Lichtenstein mesh hernioplasty. In our study, there was only 1 short term recurrence that was noted during follow up in OPD in the Laparoscopy group and in the Open Lichtenstein group.

\section{Conclusion}

The various benefits of TEP technique found in the current study include lesser post-operative pain, less analgesic requirement, shorter hospital stay, shorter sick leave. Limitations of the TEP technique include longer operative time, longer learning curve, higher conversion rates to open surgery, greater incidence of intraoperative complications and the need for general anaesthesia for the surgery. We recommend that a surgeon should use this laparoscopic technique for hernia repair after knowing about all the pros and cons of the technique, his expertise for the technique and the infrastructure available.

\section{Conflicts of Interest: None}

\section{Source of Funding: Self}

Ethical Clearance: Ethical clearance has been taken from the ethical committee.

\section{Bibliography}

1. Fasih T, Mahapatra TK, Waddington RJ. Early results of inguinal hernia repair by the 'mesh plug' technique. Ann R Coll Surg Engl 2000; 82 (6): 396400 .

2. Franciosi C, Romano F, Caprotti R, De Fina S, Colombo G, Visintini G, Sartori P, Uggeri F. Hernia repair with Prolene mesh according to Lichtenstein technique. Minerva Chir 2000; 55(9): 593-97.

3. Epidemiological, Economic and Sociologic aspects of hernia surgery in the United States in the 1990s; Ira M. Rutkow, Surgical Clinics of North America, Vol. 78, No. 6, December 1998, pp - 941-951.

4. Gross J, Synder S. A critical view of inguinal hernia. Surg Clin In, 1953.

5. Stoker D, Spiegelhalter D, Sing R, et al. Laparoscopic versus open inguinal hernia repair: randomized prospective trial. Lancet 1994; 343: 1243-1245.

6. Liem MSL, van der Graaf Y, van Steensel CJ, et al. Comparison of conventional anterior surgery and laparoscopic surgery for inguinal-hernia repair. $\mathrm{N}$ 
Engl J Med 1997; 336: 1541-7.

7. Neumayer L, Giobbie-Hurder A, Jonasson O, et al. Open mesh versus laparoscopic mesh repair of inguinal hernia. N Engl J Med 2004;350(18):18191827 [PubMed: 15107485]

8. Felix E, Habertson N, Varteian S. Laparoscopic hernioplasty: significant complications. Surg Endosc 1999; 13: 328-331.

9. Tedik C, Arregui M, Dulucq J, et al. Complications and recurrences with laparoscopic repair of groin hernias. A multi-institutional retrospective analysis. Surg Endosc 1994; 8:1316-1323.

10. MacFadyen B, Mathis C. Inguinal herniorrhaphy; complications and recurrences. Semin Lap Surg 1994;1(2): 128-140.
11. Felix E, Michas C, Gonzalez M. TAPP vs TEP laparoscopic hernioplasty. Surg Endosc 1995; 9:984-989.

12. Lal P, Kajla RK, Chander J, Saha R, Ramteke VK. Randomized controlled study of laparoscopic total extraperitoneal versus open Lichtenstein inguinal hernia repair. Surg Endosc 2003;17(6):850-856 [PubMed: 12658428]

13. Wennstrom I, Berggren P, Akerud L, Jarhult J. Equal results with laparoscopic and Shouldice repairs of primary inguinal hernia in men. Report from a prospective randomised study. Scand J Surg 2004;93(1):34-36 [PubMed: 15116817] 